ORIGINAL ARTICLE

\title{
Evaluation of Parasitological Methods for the Detection of Strongyloides Stercoralis among Individuals in Selected Health Institutions In Addis Ababa, Ethiopia
}

\author{
Tamirat Hailegebriel $^{1}$, Beyene Petros $^{2}$, Tekola Endeshaw ${ }^{3}$
}

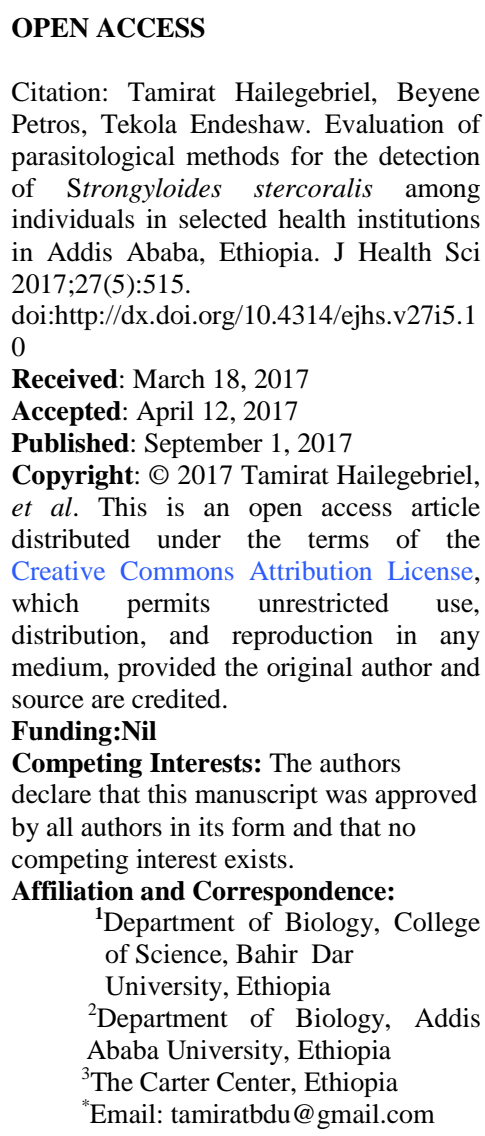

OPEN ACCESS

Citation: Tamirat Hailegebriel, Beyene Petros, Tekola Endeshaw. Evaluation of parasitological methods for the detection of Strongyloides stercoralis among individuals in selected health institutions in Addis Ababa, Ethiopia. J Health Sci 2017;27(5):515.

doi:http://dx.doi.org/10.4314/ejhs.v27i5.1 0

Received: March 18, 2017

Accepted: April 12, 2017

Published: September 1, 2017

Copyright: (๑) 2017 Tamirat Hailegebriel, et al. This is an open access article distributed under the terms of the Creative Commons Attribution License, which permits unrestricted use, distribution, and reproduction in any medium, provided the original author and source are credited.

Funding:Nil

Competing Interests: The authors declare that this manuscript was approved by all authors in its form and that no competing interest exists.

Affiliation and Correspondence: ${ }^{1}$ Department of Biology, College of Science, Bahir Dar University, Ethiopia ${ }^{2}$ Department of Biology, Addis Ababa University, Ethiopia ${ }^{3}$ The Carter Center, Ethiopia "Email: tamiratbdu@gmail.com

\section{ABSTRACT}

BACKGROUND: Strongyloides stercoralis causes chronic intestinal infection that may lead to disseminated disease during immunosuppression. Therefore, efficient diagnostic methods are essential to detect silent and latent infections. The present study was aimed to compare the efficacy of parasitological methods for the detection of $S$. stercoralis infection.

METHODS: A cross-sectional study was conducted on 351 subjects (226 HIV positive and $125 \mathrm{HIV}$ negative) from October 2005 to May 2006. The study participants were selected by systematic random sampling method. Fresh fecal samples were collected from each participant and processed by different parasitological methods.

RESULTS: Among the 351 individuals involved in the study, 43(12.3\%) were infected by $S$. stercoralis. The prevalence of $S$. stercoralis infection was significantly higher among HIV positive subjects (17.3\%) as compared with $3.2 \%$ in HIV negative subjects $(p=0.001)$. The odd of being infected by $S$. stercoralis was significantly higher among AIDS patients $(O R=6.31$; 95\% CI, 2.218.1) and HIV patients with diarrhea (OR=9.3; 95\% CI 4.6-18.9) as compared with respective controls. Agar plate culture showed superior sensitivity (97.7\%) than other methods for detection of $S$. stercoralis. Similarly, agar plate culture showed strong diagnostic agreement with Baermann's method (kappa $=0.82$; 95\% CI 0.72 0.92 ) followed by fecal concentration (kappa $=0.67 ; 95 \% \mathrm{CI}=0.54$ $0.80)$.

COCLUSION: This study revealed that HIV patients were about 6 folds susceptible for S. stercoralis infection. Similarly, agar plate culture showed superior diagnostic efficiency for detections of $S$. stercoralis infection.

KEYWORDS: S. stercoralis, HIV positive, agar plate culture

\section{INTRODUCTION}

Strongyloidiasis is an intestinal infection of humans and other primates mainly caused by Strongyloides stercoralis, which is predominant in South East Asia, Latin America and sub-Saharan 
Africa (1). S. stercoralis is currently infecting 50 to 100 million people worldwide (2). Even though the infection commonly occurs in humans, sometimes other primates such as dogs, cats and Mongolian gerbils are also reported (3).

S. stercoralis infection usually results in asymptomatic infection of the gastrointestinal tract and remains undetected for decades in most "healthy infected" individuals (4). However, it may result in a wide range of complaints during immunosuppression due to various factors, including in HIV patients and individuals under immunosuppressive drug therapy (5).

In addition to the well-known opportunistic intestinal parasites (Cryptosporidium parvum, Isospora belli, Cyclospora caytanesis and microsporidium species), $S$. stercoralis is also very common among HIV patients in Ethiopia $(6,7)$. The infection with $S$. stercoralis usually causes chronic diarrhea with a wide variety of intestinal and extra-intestinal problems. Sometimes, the infection results in hyperinfection syndrome and is disseminated to ectopic sites such as brain, kidney and liver when the individual is severely immunocompromised. The mortality rate due to $S$. stercoralis hyperinfection may be as high as $87 \%$ in the presence of secondary bacterial infection $(8,9)$ and may reach up to $90 \%$ among HIV patients (10).

$S$. stercoralis is one of the most difficult helminthic infections to diagnosis especially in chronic cases with minimal and irregular larval output. Although different parasitological methods are available for the detection of this infection, the detection rates of the methods are very low and even may lead to false negative results. Now a days, agar plate is widely applied in most endemic countries with a higher detection rate than other parasitological methods. According to previous reports, the detection rate of agar plate culture may reach up to $70 \%$ from single stool specimens (11) and may increase up to $96 \%$ when three serial stool samples are taken $(8,12)$. However, agar plate culture has not been used for the detection of $S$. stercoralis in Ethiopia. Therefore, ths study was aimed to evaluate the efficacy of different parasitological methods for the detection of
Strongyloides and to determine the prevalence of $S$. stercoralis infection.

\section{MATERIALS AND METHODS}

Study population and design: A cross-sectional study was conducted from October 2005 to May, 2006 among HIV positive and HIV negative subjects in selected health institutes in Addis Ababa. The study subjects were selected by systematic random sampling methods when they visited voluntarily counseling and testing (VCT) centers for HIV. The serological test for HIV antibody test was done by WHO guidelines within the selected health institutions (MiniliK II Hospital, Yekatit 12 Hospital, Zewoditu Hospitals and Kerkos Health Center) by trained health professionals.

The sample size was determined by statistical formula $\mathrm{n}=\mathrm{Z}^{2} \mathrm{xP}(1-\mathrm{P}) / \mathrm{d}^{2}: \mathrm{P}$ (prevalence of $S$. stercoralis infection), $\mathrm{d}$ (at 5\% marginal error) and standard score (Z) at $95 \%$ confidence interval. The average prevalence of $S$. stercoralis infection in the area is not known. Thus, the prevalence of $S$. stercoralis was assumed 50\%. Therefore, the sample size was 384 study subjects.

The prevalence of $S$. stercoralis is very low in the general population as compared with immunocompromized subjects such as HIV/AIDS patients. The objective of this study was to compare the efficacy of parasitological methods for detection of $S$. stercoralis. A large number of $S$. stercoralis positive samples are needed to compare efficacy of parasitological methods. Thus, a large number of HIV positive subjects were invited to the study as compared with HIV negative control groups.

Ethical consideration: The project was ethically cleared by the Ethical Committee of Biology Department, Addis Ababa University, and the Addis Ababa City Administration Health Bureau. After informing the study subjects about the benefit of the study, individuals who volunteered to participate and gave their written consents were included in the study. All the results were kept strictly confidential, and individuals who were positive for intestinal parasites were treated free of charge.

DOI: http://dx.doi.org/10.4314/ejhs.v27i5.10 
Stool examination: All stool samples were processed within 2 to 3 hours of collection in the parasitological laboratory of the former Ethiopian Health and Nutrition Research Institute (EHNRI). The stool specimens were processed by different parasitological methods described below.

Agar plate culture: The stool samples were processed using standard protocol as described previously (11). Briefly, about 4 grams of feces were placed at the center of nutrient agar plate, and the edge of the plate was sealed with adhesive tape to prevent the migration of larvae to outside surface. The plates were incubated for 2 to 5 days at room temperature and observed daily with inverted microscope for the presence of tracks, due to moving larvae or free-living adults. At the $5^{\text {th }}$ day, $5 \mathrm{ml}$ of $10 \%$ formalin solution was added to the plates and kept for five minutes. The formalin solution was transferred into $15 \mathrm{ml}$ test tubes and centrifuged at 1500 revolution per minute (rpm) for two minutes. The sediments were analyzed microscopically for the presence of $S$. stercoralis filariform larvae, adults and/or eggs.

Baermann's method: Fecal specimens were processed using the standard method described previously (8). Five gram of fresh feces was placed at the center of sieve mesh, which was partly immersed in a sedimentation flask containing warm water at $37^{\circ} \mathrm{C}$. The fecal specimens were left for one hour at room temperature, which makes the larvae crawl out of the fecal suspension and migrate to warm water. The upper portion of the water was discarded by retaining $10 \mathrm{ml}$ at the bottom of the funnels. The remaining fluid was transferred to a $15 \mathrm{ml}$ test tube and centrifuged at $5000 \mathrm{rpm}$ for 5 minutes. The sediment was examined microscopically for the presence of larvae.

Formal ether concentration: The stool specimens were processed by standard protocol used for detection of parasitic organism as described before (13). The presences of $S$. stercoralis larvae or eggs were examined microscopically.

Direct fecal smear: About $2 \mathrm{mg}$ of fresh stool sample was thoroughly mixed with a drop of normal saline on a glass slide. The preparation was covered with cover slip and examined microscopically for the presence of S. stercoralis first stage (L1) larvae.

Harada Mori filter paper: Filter paper containing fresh fecal material was placed in a test tube containing water and incubated for 13 days at $28^{0} \mathrm{C}$. The amount of water in the tube was checked every day and replaced before dried. On the $13^{\text {th }}$ day, the filter paper with stool sample was removed, and the test tube containing the water was centrifuged for 5 minutes at $2000 \mathrm{rpm}$. The sediments were examined under microscope using $10 \mathrm{x}$ and $40 \mathrm{x}$ objectives for the presence of $S$. stercoralis filariform larvae (8).

Charcoal culture: Ten gram of fresh fecal materials was thoroughly mixed with distilled water and then mixed with equal quantity of granulated charcoal. The fecal-charcoal mixtures were placed at the center of petri-dish which was covered with moisten filter paper at the bottom. The petri-dish was placed in a dark room after being sealed with vinyl tape. The petri-dish was checked every day, and the evaporated water was replaced up to 13 days. On the $13^{\text {th }}$ day, water was added to the mixture and exposed to light for 2 hours. The water was collected in a test tube and centrifuged for 5 minutes at $2000 \mathrm{rpm}$. The sediments were examined microscopically for the presence of S. stercoralis larvae.

Data Analysis: The collected data were properly recorded and analyzed using statistical package for social science (SPSS) version 20.0. Binary logistic regression analysis was performed to assess the association of $S$. stercoralis infection with HIV status, diarrheal status and other demographic variables. The strength of association was determined by Chi-square test and odd ratio. A defined value of $\mathrm{p}<0.05$ was considered as significant. The degrees of agreement between parasitological methods were evaluated by Kappa statistics. The comparisons of agar plate method with other parasitological methods were performed by Fisher's exact test.

\section{RESULTS}

The response rate of study participants was $91.4 \%$. Among 384 subjects invited for the study, 351 individuals (226 HIV positive and 125 HIV negative) were participated in the study by

DOI: http://dx.doi.org/10.4314/ejhs.v27i5.10 
providing stool specimens and other personal information. The age range of the study participants was 15 to 65 years with a mean age of 30.2 years. The majority of the study populations were female: $65.9 \%$ among HIV positive and $64.8 \%$ among HIV negative subjects.

The overall prevalence of intestinal parasites among study participants was 35\% (37.2\% among HIV positive and $31.2 \%$ HIV negative individuals). The prevalence of intestinal parasites was even higher among HIV positive subjects with chronic diarrhea (49.6\%) as compared with $28.9 \%$ among HIV positive subjects without diarrhea. The most prevalent intestinal parasites detected from study participants were $S$. stercoralis $(12.3 \%)$ and E. histolytica/dispar (Table 1). The prevalence of $S$. stercoralis infection was significantly higher among HIV positive subjects as compared with HIV negative subjects $(\mathrm{p}=0.001)$.

Table 1: Prevalence of intestinal parasites detected among HIV positive and HIV negative subjects in selected health institutions in Addis Ababa, 2005/2006.

\begin{tabular}{|c|c|c|c|c|}
\hline \multirow[t]{2}{*}{ Parasites detected } & \multicolumn{3}{|c|}{ HIV status } & \multirow[b]{2}{*}{$\begin{array}{l}\text { Chi-square } \\
\text { p- value }\end{array}$} \\
\hline & $\begin{array}{l}\text { HIV positive }(\mathrm{n}=226) \\
\text { No. }(\%)\end{array}$ & $\begin{array}{l}\text { HIV negative }(\mathrm{n}=125) \\
\text { No. }(\%)\end{array}$ & $\begin{array}{l}\text { Total }(n=351) \\
\text { No. }(\%)\end{array}$ & \\
\hline \multicolumn{5}{|l|}{ Helminths } \\
\hline Strongyloides stercoralis & $39(17.3)$ & (3.2) & $43(12.3)$ & $0.001 *$ \\
\hline Hookworm species & $5 \quad(2.2)$ & $7 \quad(5.6)$ & $12(3.4)$ & 0.290 \\
\hline Ascaris lumbricoides & $11(4.9)$ & $10(8)$ & $21(6)$ & 0.236 \\
\hline Trichuris trichiura & $4 \quad(1.8)$ & 0 & $4 \quad(1.1)$ & 0.196 \\
\hline Hymenolepis dimunta & $1 \quad(0.4$ & 0 & $1 \quad(0.3)$ & 0.456 \\
\hline Hymenolepis nana & 0 & $(0.8)$ & $1 \quad(0.3)$ & 0.178 \\
\hline \multicolumn{5}{|l|}{ Protozoan } \\
\hline E. histolytica/dispar & $20(8.8)$ & $10(8)$ & $30(8.6)$ & 0.502 \\
\hline Entamoeba coli & 3 (1.3) & $5 \quad(4)$ & $8 \quad(2.3)$ & 0.108 \\
\hline Endolimax nana & $1 \quad(0.4)$ & 0 & $1 \quad(0.3)$ & 0.456 \\
\hline Giardia lamblia & (3.5) & (2.4) & $11(3.1)$ & 0.557 \\
\hline Chilomastix mesnili & 0 & $(0.8)$ & $1 \quad(0.3)$ & 0.456 \\
\hline Blastocystis hominis & $14(6.2)$ & (6.4) & $21(6)$ & 0.822 \\
\hline Sarcocyst species & 0 & $1 \quad(0.8)$ & $1 \quad(0.3)$ & 0.178 \\
\hline Total infected & $84 \quad(37.23)$ & $39 \quad(31.2)$ & $123(35)$ & \\
\hline
\end{tabular}

Out of the $43 \mathrm{~S}$. stercoralis positive result, 39(90.3\%) were HIV positive while the remaining 4 (9.7\%) were HIV negative subjects. The likelyhood of being infected by $S$. stercoralis was increase 6 folds among HIV positive subjects as compared with HIV negative subjects $(\mathrm{OR}=6.31$; 95\% CI, 2.20 - 18.10). Similarly, the risk of $S$. stercoralis infection was even increased by 9 folds among HIV patients with chronic diarrhea as compared with HIV positive subjects without diarrhea $(\mathrm{OR}=9.34 ; 95 \% \mathrm{CI}, 4.60$ - 18.98) (Table 2 ). These showed that HIV positive subjects were susceptible to $S$. stercoralis infection.

Out of the total study participants, 43 individuals were positive for $S$. stercoralis by at least one of the six parasitological methods used in the study. The sensitivity of the different parasitological methods was evaluated for the detection of $S$. stercoralis. Among the methods evaluated in the study, agar plate culture showed the highest sensitivity $(97.7 \%)$ followed by Baermann's method (73.8\%) while charcoal culture showed the lowest sensitivity $(38.1 \%)$ for detection of $S$. stercoralis infection (Table 3). The contribution of agar plate culture for detection of $S$. stercoralis was significantly higher as compared with charcoal and Harada Mori filter paper culture $(\mathrm{p}=0.000)$.

The agreements of different parasitological methods for the detection of S. stercoralis were

DOI: http://dx.doi.org/10.4314/ejhs.v27i5.10 
evaluated using kappa (k) statistic. Agar plate culture showed strong diagnostic agreement with Baermann's method $(\mathrm{k}=0.82 ; 95 \% \mathrm{CI}=0.72$ to $0.92)$ followed by formal ether concentration $(\mathrm{k}=$ $0.67 ; 95 \% \mathrm{CI}=0.54$ to 0.80 ). Both diagnostic methods showed high detection rate of $S$. stercoralis. On the other hand, agar plate culture showed moderate agreement with Harada Mori filter paper $(\mathrm{k}=0.59)$ and charcoal culture $(\mathrm{k}=$ 0.51) (Table 3). Harada Mori filter paper and charcoal culture failed to detect more than $50 \%$ of the $S$. stercoralis positive results.

Table 2: HIV status and sociodemographic factors associated with S. stercoralis infection in selected health institutes in Addis Ababa, Ethiopia, 2005/2006.

\begin{tabular}{|c|c|c|c|c|c|c|}
\hline \multirow[t]{2}{*}{ Parameters } & & \multicolumn{3}{|c|}{ S. stercoralis infection } & \multirow{2}{*}{$\begin{array}{l}\text { Crud OR } \quad(95 \% \\
\text { CI) }\end{array}$} & \multirow[t]{2}{*}{ P-value } \\
\hline & & Positive (\%) & Negative (\%) & Total (\%) & & \\
\hline \multirow[t]{2}{*}{ Sex } & Female & $27(7.7)$ & $201(57.3)$ & $228(65)$ & $1.11(0.58-2.16$ & 0.751 \\
\hline & Male & $16(4.6)$ & $107(30.5)$ & $123(35)$ & 1.00 & \\
\hline \multirow[t]{4}{*}{ Age } & $15-24$ & $10(2.8)$ & $84(23.9)$ & $94(26.8)$ & $1.16(0.34-3.98)$ & 0.815 \\
\hline & $25-34$ & $18(5.1)$ & $116(33)$ & $134(38.2)$ & $0.89(0.28-2.83)$ & 0.842 \\
\hline & $35-44$ & $11(3.1)$ & $79(22.5)$ & $90(25.6)$ & $0.99(0.29-3.36)$ & 0.988 \\
\hline & $>44$ & $4(1.1)$ & $29(8.3)$ & $33(9.4)$ & 1.00 & \\
\hline \multirow[t]{2}{*}{ HIV status } & Positive & $39(11.1)$ & $187(53.3)$ & $226(64.4)$ & $6.31(2.20-18.10)$ & $0.001 *$ \\
\hline & Negative & $4(1.1)$ & $121(34.5)$ & $125(35.6)$ & 1.00 & \\
\hline \multirow[t]{2}{*}{ Diarrhea } & Yes & $30(8.5)$ & $61(17.4)$ & $91(25.9)$ & $9.34(4.60-18.98)$ & $0.000 *$ \\
\hline & No & $13(3.7)$ & $247(70.4)$ & $260(74.1)$ & 1.00 & \\
\hline
\end{tabular}

The free-living adults were observed after five days of culturing in agar plate culture. In some cases, the eggs were hatched and the rhabditiform larvae were clearly visible inside the body of adult female worms after keeping the plate for seven days which shows $S$. stericoralis is not only oviparous but also it is larviparous (Fig. 1). In addition, all stages of the life cycles (eggs, rhabditiform larvae, filariform larvae and adult stage) were observed in the present study from a single agar plate culture after keeping the plate for seven days at room temperature (Fig. 2).
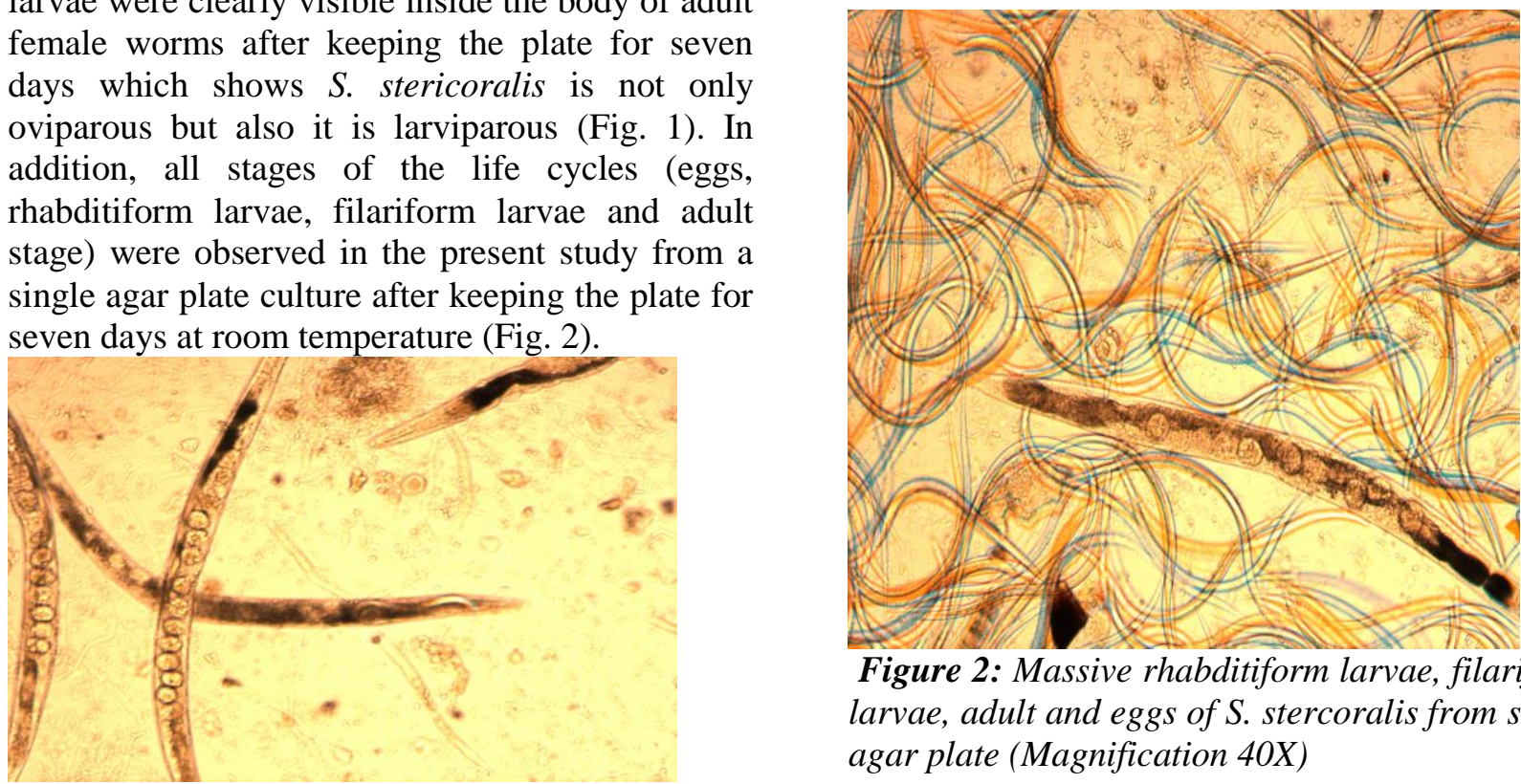

Figure 2: Massive rhabditiform larvae, filariform larvae, adult and eggs of $S$. stercoralis from single agar plate (Magnification 40X)

Figure 1: Adults female S. stercoralis worm with eggs and rhabiditiform Larvae obtained from agar plate (Magnification 40X)

DOI: http://dx.doi.org/10.4314/ejhs.v27i5.10 
Table 3: Comparison and agreement of agar plate culture with other parasitological methods for the detection of $S$. stercoralis infection (43 positive results from 351 subjects)

\begin{tabular}{|c|c|c|c|c|c|c|c|}
\hline \multirow[t]{2}{*}{$\begin{array}{l}\text { Parasitological } \\
\text { methods }\end{array}$} & \multicolumn{5}{|c|}{ Agar plate culture $* *$} & \multicolumn{2}{|c|}{$\begin{array}{l}\text { Degree of agreement } \\
\text { between methods } \dagger\end{array}$} \\
\hline & Positive & Negative & Total & $\begin{array}{l}\text { Sensitivity } \\
(\%)\end{array}$ & $\begin{array}{l}\text { Specificity } \\
(\%)\end{array}$ & Kappa & $95 \% \mathrm{CI}$ \\
\hline \multicolumn{8}{|l|}{ Baermann's method } \\
\hline Positive & 31 & 1 & 32 & 73.8 & 99.7 & 0.819 & $0.719-0.919$ \\
\hline Negative & 11 & 308 & 319 & & & & \\
\hline Total & 42 & 309 & 351 & & & & \\
\hline \multicolumn{8}{|l|}{ Charcoal culture } \\
\hline Positive & 16 & 1 & 17 & 38.1 & 99.7 & 0.508 & $0.351-0.665$ \\
\hline Negative & 26 & 308 & 334 & & & & \\
\hline Total & 42 & 309 & 351 & & & & \\
\hline \multicolumn{8}{|l|}{ Direct fecal smear } \\
\hline Positive & 21 & 1 & 22 & 50 & 99.7 & 0.625 & $0.484-0.766$ \\
\hline Negative & 21 & 308 & 329 & & & & \\
\hline Total & 42 & 309 & 351 & & & & \\
\hline \multicolumn{8}{|c|}{ Formal ether-concentration } \\
\hline Positive & 23 & 1 & 24 & 54.8 & 99.7 & 0.668 & $0.535-0.801$ \\
\hline Negative & 19 & 308 & 327 & & & & \\
\hline Total & 42 & 309 & 351 & & & & \\
\hline \multicolumn{8}{|c|}{ Harada Mori Filter paper } \\
\hline Positive & 19 & 0 & 19 & 45.2 & 100 & 0.593 & $0.446-0.740$ \\
\hline Negative & 23 & 309 & 332 & & & & \\
\hline Total & 42 & 309 & 351 & & & & \\
\hline
\end{tabular}

\section{DISCUSSION}

Strongyloides stercoralis is the predominant intestinal helminth frequently identified from HIV positive subjects with chronic diarrhea in the tropics $(14,15)$. The result of this study showed that $S$. stercoralis infection was highly prevalent among HIV patients than among HIV negative subjects. This finding is in line with other works reported from Ethiopia $(6,7)$ and from Brazil $(11,16)$. The prevalence of $S$. stercoralis infection among HIV patients in this study was a bit higher than $3.4 \%$ to $13 \%(6,17,18)$ reported from different parts of Ethiopia. The high prevalence of $S$. stercoralis infection in this study could be due to the fact that agar plate culture has superior detection rate than the parasitological methods used in others studies.
The likelihood of being infected by $S$. stercoralis was increased by 6 folds in HIV positive subjects as compared with HIV negative subjects in this study. This finding is in line with 6 fold increase susceptibility of HIV positive subjects for $S$. stercoralis infection reported elsewhere $(11,19)$. This study revealed that the larval output was significantly higher in HIV patients as compared with HIV negative subject, which is in agreement with a previous report (6).

The detection of $S$. stercoralis infection in most clinical settings of Ethiopia is under estimated either due to minimal larval output with feces or poor sensitivity of parasitological methods. Thus, appropriate diagnosis has to be in place in order to detect chronic cases that might

DOI: http://dx.doi.org/10.4314/ejhs.v27i5.10 
lead to fulminate when the immune status of the individual is compromised. Although agar plate culture had higher sensitivity for detection of $S$. stercoralis, it was not implemented in Ethiopia.

In this study, six different parasitological methods were compared to detect $S$. stercoralis infection. The sensitivity of agar plate culture in this study was $97.7 \%$, which is in line with 95$96 \%$ reported elsewhere $(8,20)$. Baermann's diagnostic method showed moderate sensitivity $(73.8 \%)$ to detect $S$. stercoralis infection, which is in agreement with $72.3 \%$ reported elsewhere (21). The sensitivity of fecal concentration in this study was $54.8 \%$, which is a bit high as compared with 27.5\% reported elsewhere (21). Direct fecal smear, Harada Mori filter paper and charcoal method showed poor sensitivity while agar plate culture showed higher sensitivity for the detection of S. stercoralis.

Cohen's Kappa statistics showed that agar plate culture and Baermann's method have strong diagnostic agreement. At least $75 \%$ of $S$. stercoralis infection was detected by either agar plate culture or Baermann's methods. Agar plate was about 24\% more efficient than Baermann's method in this study, which is in agreement with other reports (22). This study revealed that agar plate culture was nearly 2 folds more efficient to detect $S$. stercoralis infection than direct fecal smear, formal-ether concentration and fecal culture using charcoal and Harada Mori filter paper. Similar high efficiency of agar plate culture from direct fecal smear and formal-ether concentration was reported elsewhere (21).

The higher detection rate of $S$. stercoralis larvae with agar plate culture could be explained by the detection of free living stage of the parasite following the life cycle compared with other parasitological methods. This may be due to the fact that agar plate culture supports the switching of developmental stages from parasitic life to freeliving stage. The agar plate culture has other advantages such as harvesting all stages of the lifecycle from a single plate which is important for further detailed studies and obtaining a large number of filariform larvae for antigen preparation.
In conclusion, this study showed that $S$. stercoralis is the most prevalent intestinal parasite among HIV positive subjects. This suggests that HIV positive subjects are more susceptible for $S$. stercoralis infection. In addition, this study revealed that agar plate culture is a superior diagnostic method for the detection of $S$. stercoralis to other parasitological methods. However, the choice of parasitological methods for routine laboratory work takes into account sensitivity, cost and time. Although the agar plate is superior to other parasitological methods, it may not be used for routine laboratory work due to its cost and prolonged culturing time. Therefore, we recommend this method for immunocompromised subjects especially with HIV and patients receiving immunosuppressive drug therapy, suspected for S. stercoralis infection and generally for epidemiological and in-depth research activities.

\section{ACKNOWLEDGMENTS}

We would like to thank all study participants for providing samples and personal information for the study. We would like to forward our gratitude to health professionals and management staff of the selected health institutions. Finally, we forward our appreciation to the former Ethiopian Health and Nutrition Research Institute (EHNRI) for allowibg us to work in their laboratory.

\section{REFERENCES}

1. Lia C, Hsu Y, Wang J, Lin C. Infection with Bloody Pericardial Effusion in a Non-Immunosuppressed Patient. Circ J. 2002;66:613-4.

2. Marty FM, Lowry CM, Rodriguez M, Milner DA, Pieciak WS, Sinha A, et al. Treatment of Human Disseminated Strongyloidiasis with a Parenteral Veterinary Formulation of Ivermectin. Clin Infect Dis. 2005;41:e5-8.

3. Stephenson LS, Latham MC, Ottesen EA Malnutrition and parasitic helminth infections. Parastol. 2000;121:S23-S38.

4. Dada-Adegbola HO, Bakare RA. Strongyloidiasis in children five years and

DOI: http://dx.doi.org/10.4314/ejhs.v27i5.10 
below. West Africa J Med. 2004;23(3):194-7.

5. Nonaka D, Takaki K, Tanaka M, Umeno M, Takeda T, Yoshida M, et al. Paralytic ileus due to Strongyloidiasis: case report and review of the literature. Am J Trop Med Hyg. 1998;59(4):535-8.

6. Awole M, Gebre-Selassie S, Kassa T, Kibru G. Prevalence of Intestinal Parasites in HIV-Infected adult Patients in Southwestern Ethiopia. Ethiop J Health Dev. 2003;17(1):71-8.

7. Endeshaw $\mathrm{T}, \quad$ Mohamoud $\mathrm{H}$, Woldemicheal T. C. parvum and other intestinal parasites among diarrheal patients referred to EHNRI in Ethiopia. Ethiop Med J. 2004;42:195-8.

8. Siddiqui AA, Berk SL. Diagnosis of Strongyloides stercoralis Infection. Clin Infect Dis. 2001;33:1040-7.

9. Johnston FH, Morris PS, Speare R, McCarthy J, Currie B, Ewald D, et al. Strongyloidiasis: A review of the evidence for Australian practitioners. Aust J Rural Health. 2005;13:247-54.

10. Hammad G, Lenox RJ. Strongyloidiasis in immunocompromised patients: Case Report. The Guthrie J. 1999;68(2):65-8.

11. Blatt JM, Cantos GA. Evaluations of techniques for the diagnosis of Strongyloides stercoralis in human immunodeficiency Virus (HIV) positive and HIV negative individuals in the city of Itajai, Brazil. Brazilian. J Infect Dis. 2003;7(6):1-7.

12. Moustafa MA. An evaluation of the modified agar plate method for the diagnosis of Strongyloides stercoralis. Egypt Soc Parasitol. 1997;27(2):571-9.

13. Cheesbrough M. Medical Laboratory Manual for Tropical Countries. HIV Suppl. 1990:S2-S23.

14. Simon K. Gastrointestinal tract diseases in HIV-infected subjects with AIDS. HIV /AIDS Rev. 2002;2(2):1-5.
15. Sathiyasekaran $M$, Shivbalan S. Intestinal Strongyloidiasis and Common Variable Immuno-Deficiency Syndrome. Indian Pedia. 2005;42:17.

16. Cimerman S, Cimerma B, Lewi DS. Enteric parasites and AIDS. Sao Paulo Med J Rev Paulo Med. 1999;117(6):26673.

17. Fontanet AL, Sahlu T, Rinke-de-Wit T, Messele T, Masho W, Woldemichael T, et al. Epidemiology of infections with intestinal parasites and human immunodeficiency virus (HIV) among sugar-estate residents in Ethiopia. Ann Trop Med Parasitol. 2000;94:269-78.

18. Fisseha B, Petros B, Woldemicheal T, Mohammed H. Diarrhea associated parasitic infectious agents in AIDS patients within selected Addis Ababa Hospital. Ethiop $J$ Health Dev. 1999;13(3):169-74.

19. Getaneh A, Medhin G, Shimelis T. Cryptosporidium and Strongyloides stercoralis infections among people with and without HIV infection and efficiency of diagnostic methods for Strongyloides in Yirgalem Hospital, southern Ethiopia. BMC Research Notes 2010;3:90.

20. Montes M, Sawhney C, Barros N. Strongyloides stercoralis: there but not seen. Curr Opin Infect Dis. 2010;23(5):500-4.

21. Inêsa E, Souzaa JN, Santosa RC, Souzaa ES, Santosa FL, Silva MLS, et al. Efficacy of parasitological methods for the diagnosis of Strongyloides stercoralis and hookworm in faecal specimens. Acta Tropica 2011;120:206- 10.

22. Egido JM, Diego JA, Penin P. The Prevalence of Enteropathy due to Strongyloidiasis in Puerto Maldonado (Peruvian Amazon). The Brazilian J Infect Dis. 2001;5(3):119-23. 